\title{
Hyperreflexia in Guillain Barre Syndrome: A Case Report
}

\author{
AK SHAIKH ${ }^{\mathrm{a}}$, P SENGUPTA ${ }^{\mathrm{b}}$, KD MOHAMMAD ${ }^{\mathrm{c}}, \mathrm{SM} \mathrm{ALAM}^{\mathrm{d}}$, \\ R RABBANI ${ }^{\mathrm{e}}, \mathrm{M} \mathrm{HOSSAIN}^{\mathrm{f}}$, MK DASg
}

\begin{abstract}
:
Guillain Barre Syndrome (GBS) is an immune mediated polyradiculoneuropathy classically characterized by acute ascending type of motor weakness of limbs with areflexia but in AMAN variant of GBS preserved or exaggerated reflex sometimes can occur. We report a 45 year old female patient who presented with acute flaccid quadriplegia, bilateral facial lower motor type nerve palsy and bulbar involvement, initial hyperreflexia of all four limbs and bilateral plantar extensor response 7 days following an attack of diarrhoea. Sensory and bowel bladder function was intact. She was treated with IV immunoglobulin and

Introduction:

Guillain Barre Syndrome (GBS) is an acute, frequently severe and fulminant immune mediated polyradiculopathy. ${ }^{1}$ It is clinically characterized by acute symmetric muscle weakness and areflexia or hyporeflexia. Hyperreflexia has also been reported in different GBS variants most commonly with AMAN variant. So even though hyporeflexia or areflexia is necessary for the diagnosis of GBS, hyperreflexia does not exclude a GBS variant.
\end{abstract}

a. Dr Abdul Kader Shaikh, Associate Professor, Dept. of Neuromedicine, BSMMU, Part time Consultant, Square Hospital Ltd

b. Dr Poly Sengupta, MCPS Specialist, Square Hospital Ltd, Dhaka

c. Prof Dr Kazi Deen Mohammad, Professor and Principal, Dhaka Medical College and Hospital

d. Dr Sheikh Mahbub Alam, Assistant Professor, Dept. of Neuromedicine, BSMMU, part time Consultant, Square Hospital Ltd

e. Dr Raihan Rabbani, Assosiate consultant, Square Hospital, Dhaka

f. Dr Mosharof Hossain, Specialist, Square Hospital Ltd, Dhaka

g. Dr Mrinal Kanti Das, Assistant Registrar, Mymensingh Medical College Hospital.

Address of Correspondence: Dr Abdul Kader Shaikh, Associate Professor, Dept. of Neuromedicine, BSMMU, Part time Consultant, Square Hospital Ltd

Received: 4 June, 2013

Accepted: 14 March, 2014
IV methylprednisolone. Nerve Conduction study (NCS) revealed AMAN variant of GBS. All reflexes disappeared on the $2^{\text {nd }}$ day onward but returned on $21^{\text {st }}$ day of illness when muscle power also improved. Follow up NCS confirmed regeneration of nerves of all four limbs. So in any patient presenting with acute quadriparesis GBS should be in the differential diagnosis even if there is preserved or exaggerated deep tendon reflexes.

Key words: Guillain Barre Syndrome, Hyperreflexia, AMAN variant GBS

(J Banagladesh Coll Phys Surg 2014; 32: 107-109)

We report a case of AMAN variant of GBS with brisk reflexes during the onset and recovery phase of GBS.

\section{Case report}

Mrs Shahnaj Parvin, a 45 year old female patient from Barisal was admitted in Square Hospital LTD on 19/ 03/2012 with the complains of sudden weakness of all four limbs and difficulty in swallowing of both solid and liquid diet for 1 day following an attack of diarrhea 7 days prior to admission. There was no associated fever, fatigability, headache, skin rash, diplopia, urinary incontinence, altered level of consciousness, convulsion. There was no previous history of similar weakness and no diurnal variation in weakness of limbs. She is a known case of hypertension and apart from history of hysterectomy 5 years ago she had no other significant past medical history.

On examination at admission she was fully conscious and oriented. She had bilateral lower motor neuron type facial nerve palsy and bulbar palsy with absent gag reflex. Muscle power was 3/5 in upper limbs and 1/5 in lower limbs. All deep tendon reflexes were exaggerated with bilateral extensor plantar response. She was transferred to ICU and was put on mechanical ventilation the $2^{\text {nd }}$ day of admission. One day after admission all jerks became absent except biceps jerks and muscle power in both upper and lower limbs became 1/5. On the $3^{\text {rd }}$ day all jerks were absent but plantar response continued to remain bilaterally extensor. Sensory and 
bowel bladder function was intact all through. Investigations showed normal total and differential count of WBC, electrolytes and creatinine kinase. CSF study done on $2^{\text {nd }}$ day of admission showed normal cell count with normal protein (40 mg/dl) and glucose (68 mg/dl), ADA, MTB PCR with gram stain, AFB stain and culture sensitivity were negative. MRI of brain and cervical spine were normal. NCS revealed AMAN variant of polyneuropathy. Anti Campylobacter Jejuni antibody, anti GM1 antibody was not done. She was treated with IV Immunoglobulin from Day 2 and continued for 5 days; IV methylprednisolone was given on Day 1 as cervical transverse myelitis with cranial polyneuropathy was considered as another possibility. Nine days later her muscle power started improving and 21 days after admission all jerks reappeared. At discharge she had muscle power of 2/5 in all four limbs, considerable improvement of facial nerve palsy and bulbar palsy. Follow up about 1 year later on 3/3/2013 revealed muscle power $3 / 5$ in proximal muscles of lower limb, 4/5 in distal group of muscles, 4/5 in both upper limbs. All jerks were lively, bilateral flexor plantar response. Repeat NCS revealed renervation of all four limbs which supports our diagnosis.

\section{Discussion:}

Guillain Barre Syndrome manifests as a rapidly evolving areflexic motor paralysis with or without sensory disturbance. ${ }^{1}$ Although hypo/areflexia is mandatory for the diagnosis of GBS, retained tendon reflexes or brisk reflexes have been reported in some GBS variants most commonly in AMAN in Chineese, Japanese, and Europian and Indian population. 2, 3, 4

Preceeding history of C. Jejuni infection is frequently found associated with AMAN variety of GBS with hyperreflexia. 5,6,7,8 Patients in such cases have history of abdominal pain and diarrhea. ${ }^{1}$ Hyperreflexia/ preserved tendon reflexes are thought to be present in mild or less severe form. ${ }^{2}$ Cranial, sensory, bulbar and respiratory involvement are also less frequent in such cases. ${ }^{2}$ However acute flaccid quadriplegia with bilateral facial and bulbar involvement requiring artificial ventilation on the $2^{\text {nd }}$ day of admission until 1 week before discharge in our patient indicated severe form of the disease.

Preserved tendon reflexes in GBS have been considered as an indicator of rapid clinical recovery. ${ }^{3}$ Patients with axonal GBS however can show both rapid and slow recoveries. ${ }^{5}$ It is proposed that patients with preserved tendon reflexes recover quickly because at least some of the motor units need to function to elicit visible reflexes. ${ }^{5}$ Hyperreflexia have been found to be present both in acute progressive phase and in the early recovery phase in a study, ${ }^{2}$ and also throughout the course of the illness. ${ }^{9}$ In our case, the patient had hyperreflexia of all four limbs with bilateral plantar extensor on the day of admission, however on the $2^{\text {nd }}$ day all jerks disappeared except both biceps jerks which disappeared on the $3^{\text {rd }}$ day. And all the jerks became lively 21 days after admission. Muscle power started improving on the $9^{\text {th }}$ day of admission.

Circumstantial evidence suggests that all GBS results from immune response to nonself antigens (infectious agents, vaccine) that misdirect to host nerve tissues through a resemblance of epitope (molecular mimcry) mechanism. ${ }^{1}$ A proposed mechanism for hyperreflexia is that the primary immunological attack is directed against the motor axons and Anti GM1 antibody binds with neural structures in the spinal cord as a result of the local dysfunction of the blood - CNS barrier caused by the inflammation of the spinal nerve roots leading to the dysfunction of spinal inhibitory interneurons, or of the upper motor neurons causing the abnormal reflex spread to other segments. ${ }^{2}$ Functional corticospinal tract involvement has also been proposed. ${ }^{3}$ The presence of normal sensory nerve function rather than motor is required for tendon jerks because tendon jerks are dependant on synchronized volley of impulses, a purely axonal lesion would preserve tendon jerks better than a demyelinating lesion. ${ }^{5}$

CSF shows albuminocytological dissociation in most but not all cases of GBS presenting with hyperreflexia, 2,7 and may be absent if done within $1^{\text {st }}$ week $^{1}$. We performed CSF on the $2^{\text {nd }}$ day of admission, which can explain the absence of raised protein level in our case.

Hyperreflexia is occasionally seen in chronic motor neuropathy associated with high titre anti GM1 antibody. ${ }^{2}$ Anti GM1 antibodies, anti GD 1a Ig M antibodies, Anti GalNAc GD1a Ig G antibody are also associated with AMAN variant. ${ }^{1}$ None of these could be done in our case because the tests are not available in our country.

NCS is the most important supportive investigation in the diagnosis of GBS. Hyperreflexia is more often found 
in patients with AMAN than AIDP. ${ }^{2}$ In our case the initial NCS report done on the 12th day of admission revealed AMAN variant of GBS, follow up NCS with needle EMG report revealed regeneration in all four limbs.

On the day of admission we considered it to be a case of transverse myelitis (cervical) with cranial polyneuropathy on the background of hyperreflexia and bilateral plantar extensor with bilateral lower motor neuron type facial nerve palsy along with bulbar involvement and so IV methylprednisolone was started. However subsequently all the jerks started disappearing and this led us to consider GBS.

Another possibility could be Postinfectious Myeloradiculopolyneuropathy but the neurological involvement in her case was symmetrical which goes in favour of GBS. In case of Myeloradiculopolyneuropathy neurological involvement is expected to be asymmetrical along with sensory involvement and CSF pleocytosis. GBS with stroke was not considered to explain hyperreflexia as the patient's Glassgow Coma Scale (GCS) was intact and MRI of brain was normal. Normal CSF along with normal MRI of brain excluded brainstem encephalitis to explain clinical features in our case.

\section{Conclusion:}

In conclusion, preserved tendon reflexes and even hyperreflexia may be present although rarely, in some GBS variants. So GBS should be in the differential diagnosis when dealing with any patient presenting with acute quadriparesis with preserved or brisk reflexes.

\section{References:}

1. Longo DL, Fauci AS, Braunwald E, Kasper DL, Hauser SL, Jameson JL, et al. editors. Harrison's principles of internal medicine. 18th ed. New York: McGraw Hill; 2012.

2. Kuwabara S, Ogawara K, Koga M, Mori M, Hattori T ,Yuki N. Hyperreflexia in Guillain Barre Syndrome: Relaion with acute motor axonal neuropathy and ant GM1 antibody. J Neurol Neurosurg Psychiatry.1999; 67: 180-184.

3. Singhal V, Bhat KG. Guillain Barre Syndrome with hyperreflexia: A variant. J Paediatr Neurosci. 2011;6:144-5.

4. Baheti NN, Manuel D, Shindhe PD, Radhakrishnan A, Nair M. Hyperreflexic Guillain Barre syndrome. Ann Indian Acad Neurol. 2010; 13(4): 305-307.

5. Kuwabara S, Mori M, Ogawara K, Hattori T, Yuki N. Indicators of rapid clinical recovery in Guillain Barre Syndrome. J Neurol Neurosurg Psychiatry. 2001; 70:560-2.

6. Islam Z, Jacobs BC, Belcum V, Mohammad QD, Islam MB, Herbrink P et al. Axonal variant of Guillain Barre Syndrome associated with Campylobacter infection in Bangladesh. Neurology. 2010; 74: 581-587.

7. Dhadke SV, Dhadke VN, Bangar SS, Korade MB. Clinical profile of Guillain Barre Syndrome. J Asso Physicians India. 2013; 61:168-172.

8. Selimovic BM, Lavrinc D, Mori O, Ng LK, Price L, Suturkova L et al. Enteritis caused by Campylobacter Jejuni followed by acute motor axonal neuropathy: a case report. Journal of Medical Case Reports. 2010; 4:101.

9. Yuki N, Hirata K. Preserved tendon reflexes in Campylobacter neuropathy. Ann Neurol.1998; 43:546-547. 